\title{
Long-term eribulin treatment in patients with a single kidney and retroperitoneal liposarcoma
}

\author{
Nastassja Tober*,1, Marica Gentile² \& Giuseppe lodice ${ }^{2}$ \\ ${ }^{1}$ Azienda Ospedaliero-Universitaria Policlinico Sant'Orsola-Malpighi di Bologna, Bologna, Italy \\ ${ }^{2}$ Azienda Ospedaliero-Universitaria Consorziale Policlinico di Bari, Bari, Italy \\ *Author for correspondence: nastassja@cheapnet.it
}

Retroperitoneal sarcomas are extremely rare malignant tumors. The most common type of sarcomas arising in the retroperitoneum are liposarcomas, occurring mostly in the sixth and seventh decades of life. The only potentially curative approach to liposarcomas is the complete surgical resection of the tumor with negative microscopic margins. However, retroperitoneal liposarcomas exhibit a propensity for local recurrence and distant metastasis despite the negative surgical margins, thus requiring additional therapy. Eribulin demonstrated a benefit in terms of overall survival in patients with advanced or metastatic liposarcoma. We report two cases of patients, both submitted to concomitant right nephrectomy, who experienced a long-lasting control of recurrent retroperitoneal liposarcoma before being submitted to eribulin-based therapeutic regimens ( 23 and 24 treatment cycles completed, respectively).

First draft submitted: 24 September 2019; Accepted for publication: 3 December 2019; Published online: 24 December 2019

Keywords: anthracyclines $\bullet$ eribulin $\bullet$ local recurrence $\bullet$ nephrectomy $\bullet$ retroperitoneal liposarcoma

Retroperitoneal sarcomas are extremely rare malignant tumors. The most common types of sarcomas arising in the retroperitoneum are liposarcomas, followed by leiomyosarcoma and undifferentiated pleomorphic sarcoma, formerly referred to as malignant fibrous histiocytoma [1]. Well-differentiated (lipomatous, sclerotic, and inflammatory subtype) liposarcomas are the most common variants of liposarcoma, followed by dedifferentiated liposarcomas. Of note, well-differentiated and dedifferentiated liposarcoma are the same subtype of liposarcoma (with a similar genetic profile), reflecting different grades of the tumor. Myxoid, round cell and pleomorphic liposarcomas are uncommonly found in the retroperitoneum.

Retroperitoneal liposarcomas can occur in any age group, but mostly in the sixth and seventh decades, with no clear race or sex predilection.

Retroperitoneal liposarcomas grow slowly to large sizes in the very expandable retroperitoneal space before they produce symptoms [2]. Moreover, when retroperitoneal liposarcomas produce symptoms, they are nonspecific (e.g., abdominal pain and fullness, early satiety and lower extremity swelling). Local compression or invasion of the retroperitoneal structures may also produce neurological, musculoskeletal and obstructive urinary or bowel symptoms.

Computed tomography and MRI represent the gold standard in diagnosis, staging and preoperative evaluation of liposarcomas.

Several prognostic factors for survival have been identified, including histological grade, distant and local recurrence and completeness of the surgical resection with negative margins [3].

The only potentially curative approach to liposarcomas is the complete surgical resection of the tumor with negative microscopic margins. However, retroperitoneal liposarcomas exhibit a propensity for local recurrence and distant metastasis despite the negative surgical margins, thus requiring complementary adjuvant/neoadjuvant therapy, chemotherapy or radiotherapy. The potential activities of chemotherapy include neoadjuvant cytoreductive therapy, adjuvant therapy, tumor sensitization to radiation and definitive therapy for unresectable tumors [4]. The most applied chemotherapy regimens used as first-line treatment in the setting of advanced or metastatic liposarcoma 
include doxorubicin, as single agent or in combination with ifosfamide, and gemcitabine, in combination with docetaxel [5,6]. Trabectedin and eribulin have been approved as second-line treatment in the setting of advanced or metastatic liposarcoma after first-line anthracyclines (if suitable) [7-9]. In particular, eribulin is a tubulin-targeting compound that demonstrated a benefit in terms of overall survival in patients affected by liposarcoma. In more detail, a randomized, open-label, multicenter, Phase III trial demonstrated that overall survival was improved in patients with advanced or metastatic soft-tissue sarcoma assigned to eribulin treatment compared with those assigned to dacarbazine (median 13.5 vs 11.5 months; hazard ratio: 0.77; 95\% CI: 0.62-0.95; p = 0.0169), suggesting that eribulin could be a treatment option for advanced soft-tissue sarcoma [7]. However, the occurrence of treatment-emergent adverse events was comparable in the two groups of patients ( $99 \%$ of patients who received eribulin and $97 \%$ of patients who received dacarbazine). Grade 3 or higher adverse events were more common in patients who received eribulin (67\%) than in those who received dacarbazine (56\%) [7]. Here, we describe of the cases of two patients, both submitted to surgery for liposarcoma and to right nephrectomy, who experienced a long-lasting disease control through a eribulin-based therapeutic regimen.

\section{Case reports}

\section{Case 1}

$\mathrm{SF}$ is a 68-year-old male subject with hypertension treated with ACE-inhibitors and no clinically relevant surgical history. In September 2011, he presented to the emergency room of Policlinico di Bari (Bari, Italy) with abdominal pain and a sub-occlusive episode. Abdomen MRI and CT scan showed a retroperitoneal heterogeneous mass, also consisting of fat tissue-rich areas. In October 2011, the patient underwent resection of the retroperitoneal mass and right adrenalectomy. Histopathological examination revealed a retroperitoneal well-differentiated liposarcoma (lipomatous and sclerotic subtypes), with areas of pleomorphic liposarcoma. Considering the high risk areas of liposarcoma and the positive surgical margins, the patient received post-surgical chemotherapy from December 2011 to March 2012, consisting of five cycles of epirubicin and ifosfamide (epirubicin $60 \mathrm{mg} / \mathrm{m}^{2}$ on days $1-2$ and ifosfamide $1.8 \mathrm{mg} / \mathrm{m}^{2}$ daily for 5 days, every 3 weeks) with a subsequent follow-up period.

In July 2012, a CT scan showed a retroperitoneal mass of $14 \mathrm{~cm}$ in diameter, near the right kidney, consisting mostly of fat tissue. Para-aortic and interaortocaval lymphadenopathies, maximum diameter $1.5 \mathrm{~cm}$, also affected the patient.

In November 2012, the patient underwent the resection of the recurrent retroperitoneal mass. The histopathological examination revealed a well-differentiated liposarcoma (mostly lipomatous and sclerotic subtypes, areas of inflammatory subtype), with positive surgical margins. From April 2013 to July 2013, the patient received a new line of chemotherapy, consisting of four cycles of gemcitabine $900 \mathrm{mg} / \mathrm{m}^{2}$ on days $1-8$ and docetaxel $100 \mathrm{mg} / \mathrm{m}^{2}$ on day 8 , every 21 days, with a subsequent follow-up period.

In October 2017, diagnostic imaging (ultrasound and CT scan) revealed a retroperitoneal mass, over the right kidney, of $16 \mathrm{~cm}$ in diameter with associated lombo-aortic lymphadenopathies. In April 2018, the patient was submitted to debulking surgery of the retroperitoneal mass and to right nephrectomy.

We decided to start a new line of chemotherapy in this patient, already treated with two previous lines of chemotherapy (including anthracyclines), as described above. The performance status was 1, red and white blood cell count was within the normal range and hepatic and cardiac function were normal as well. However, considering that the creatinin clearance is $<50 \mathrm{ml} / \mathrm{min}$ in a patient with a single kidney, we opted for a therapeutic regimen with lower drug doses. In May 2018, the patient started a therapy with eribulin $0.97 \mathrm{mg} / \mathrm{m}^{2}$ on days 1 and 8 of a 21-day cycle. No side effects were detected, with the exception of grade 2 constipation.

Because of grade 3 fatigue, from September 2019, we continued the treatment with a personalized schedule (eribulin $0.97 \mathrm{mg} / \mathrm{m}^{2}$ on days 1 and 15 of a 28-day cycle). In October 2018 and April 2019, CT scans showed stable disease $(16 \times 15 \mathrm{~cm}$; Figure 1). In November 2019, a CT scan showed that the tumor size increased by less than 20\% (stable disease according to the RECIST criteria). Taking into consideration radiological stable disease and clinical benefit, the patient continued the therapy with eribulin and, by now, has completed 23 treatment cycles.

\section{Case 2}

SDE is a 82-year-old male subject with paroxysmal atrial fibrillation treated with antiarrhythmic agents, hypertension treated with angiotensin receptor blockers and history of enucleation of the left eye occurred in 1966. In April 2014, the patient presented to the emergency room with abdominal pain and increasing abdominal girth. 

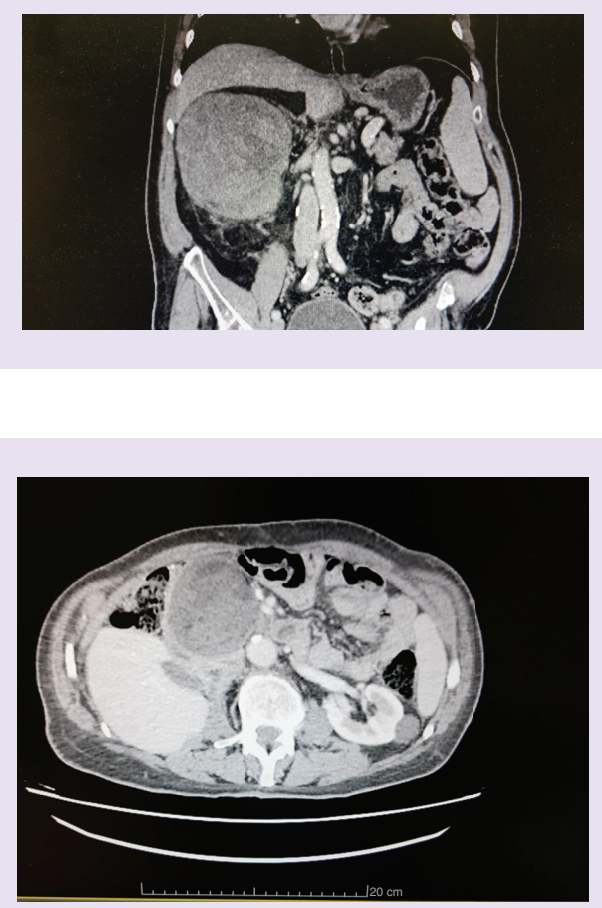

Figure 1. CT scan, October 2018, showing stable disease (a retroperitoneal mass of $16 \mathrm{~cm}$ in diameter) after six cycles of therapy with eribulin.

Figure 2. CT scan, January 2019, showing stable disease $(9.5 \times 7.5 \mathrm{~cm})$, after 11 cycles of therapy with eribulin.

Abdominal ultrasound and CT scan showed a big retroperitoneal heterogeneous mass, consisting of fluid components and fat tissue-rich areas. In June 2014, the patient was submitted to resection of the retroperitoneal mass, regional lymphadenectomy and to right nephrectomy. Histopathological examination revealed a retroperitoneal myxoid liposarcoma $(38 \times 28 \times 15 \mathrm{~cm})$, with negative nodes and surgical margins. After the surgery, the patient started a follow-up program.

In December 2017, an abdominal ultrasound showed a retroperitoneal mass of $7 \mathrm{~cm}$ in diameter. In February 2018, a positron emission tomography with radiolabelled glucose analogue 18-fluorodeoxyglucose (PET FDG) also showed a hypermetabolic mass (SUV max 3.3) at the level of L1-L4. Surgeons did not recommend surgery for this patient, so we decided to start a first line of chemotherapy. The performance status was 1 , red and white blood cell count was within the normal range and hepatic function was normal as well. However, since the creatinin clearance is $<50 \mathrm{ml} / \mathrm{min}$ in a patient with a single kidney, we opted for a therapeutic regimen with lower drug doses. Moreover, considering the cardiological comorbidities of the patient, a treatment including anthracyclines was excluded and in June 2018, the patient started a therapy with eribulin $0.97 \mathrm{mg} / \mathrm{m}^{2}$ on days 1 and 8 of a 21-day cycle. No side effects were detected, with the exception of grade 3 neutropenia (treated with granulocyte colony-stimulating factor in secondary prophylaxis) and grade 1 constipation.

In September 2018, January 2019 and October 2019, CT scans showed stable disease $(9.5 \times 7.5 \mathrm{~cm}$; Figure 2); consequently, the patient continued the therapy with eribulin and, by now, has completed 24 treatment cycles.

\section{Discussion \& conclusion}

Liposarcoma is the most frequent histological type of retroperitoneal sarcoma, a tumor that requires a more aggressive approach including, when necessary, multiorgan resection or multiple resections with recurrences. The two cases we reported here integrate the currently available literature data showing that eribulin exhibits a long-lasting control of disease in patients submitted to surgery for recurrent retroperitoneal liposarcoma and to concomitant nephrectomy, with no change in tumor size, as documented radiologically. In particular, eribulin was effective in disease control also when administered at lower doses in presence of a single kidney. Remarkably, the long-term treatment with eribulin in these nephrectomized patients was associated with no or low-grade side effects, in other words, neutropenia and constipation. These results might be useful to support the application of eribulin as first-line (case 2) or as second-line (case 1) treatment after first-line anthracyclines, in therapeutic regimens applied to patients submitted to surgery for recurrent retroperitoneal liposarcoma and to concomitant nephrectomy. 
Summary points

- The most common types of sarcomas arising in the retroperitoneum are liposarcomas.

- The mostly applied chemotherapy regimens used as first-line treatment in the setting of advanced or metastatic liposarcoma include doxorubicin, as single agent or in combination with ifosfamide, and gemcitabine, in combination with docetaxel.

- Eribulin demonstrated a benefit in terms of overall survival in patients with advanced or metastatic liposarcoma.

- Two cases of patients both submitted to surgery for liposarcoma and to right nephrectomy are reported.

- Both the patients experienced a long-lasting disease control through a eribulin-based therapeutic regimen.

- Eribulin was effective in disease control also when administered at lower doses in presence of a single kidney.

- No side effects were detected, with the exception of grade 2 constipation (case 1) and grade 3 neutropenia (case 2).

- The results support the application of eribulin treatment in patients submitted to surgery for recurrent retroperitoneal liposarcoma and to concomitant nephrectomy.

Author contributions

All authors were involved in the writing and revision of this manuscript and have provided final approval to submit.

Financial \& competing interests disclosure

The authors have no relevant affiliations or financial involvement with any organization or entity with a financial interest in or financial conflict with the subject matter or materials discussed in the manuscript. This includes employment, consultancies, honoraria, stock ownership or options, expert testimony, grants or patents received or pending, or royalties.

Medical writing support was provided by A Forte on behalf of Content Ed Net and funded by Eisai.

Ethical conduct of research

The authors state that they have obtained appropriate institutional review board approval or have followed the principles outlined in the Declaration of Helsinki for all human or animal experimental investigations. In addition, for investigations involving human subjects, informed consent has been obtained from the participants involved.

\section{References}

Papers of special note have been highlighted as: $\bullet$ of interest; $\bullet \bullet$ of considerable interest

1. Herrera-Gómez A, Ortega-Gutiérrez C, Betancourt AM, Luna-Ortiz K. Giantretroperitoneal liposarcoma. World J. Surg. Oncol. 6(1), 115 (2008).

2. Chouairy CJ, Abdul-Karim FW, MacLennan GT. Retroperitoneal liposarcoma. J. Urol. 177(3), 1145 (2007).

3. Singer S, Corson JM, Demetri GD, Healey EA, Marcus K, Eberlein TJ. Prognostic factors predictive of survival for truncal and retroperitoneal soft-tissue sarcoma. Ann. Surg. 221(2), 185-195 (1995).

4. Adarsh V, Lakshmi R. Retroperitoneal liposarcoma: a comprehensive review. Am. J. Clin. Oncol. 38(2), 213-219 (2015).

5. Maki RG. Gemcitabine and docetaxel in metastatic sarcoma: past, present, and future. Oncologist 12(8), 999-1006 (2007).

6. Katz D, Boonsirikamchai P, Choi H et al. Efficacy of first-line doxorubicin and ifosfamide in myxoid liposarcoma. Clin. Sarcoma Res. 2(1), 2 (2012).

7. Schoffski P, Chawla S, Maki RG et al. Eribulin versus dacarbazine in previously treated patients with advanced liposarcoma or leiomyosarcoma: a randomised, open-label, multicentre, Phase III trial. Lancet 387(10028), 1629-1637 (2016).

- A Phase III trial that demonstrates significant survival advantage with eribulin in patients affected by liposarcoma and leiomyosarcoma and leads to eribulin approval in advanced liposarcomas.

8. Demetri GD, von Mehren M, Jones RL et al. Efficacy and safety of trabectedin or dacarbazine for metastatic liposarcoma or leiomyosarcoma after failure of conventional chemotherapy: results of a Phase III randomized multicenter clinical trial. J. Clin. Oncol. 34(8), 786-793 (2016).

-. A Phase III trial that demonstrates a significant benefit of trabectedin versus conventional dacarbazine in advanced pretreated liposarcoma and leiomyosarcoma.

9. Constantinidou A, Jones RL. Systemic therapy in retroperitoneal sarcoma management. J. Surg. Oncol. 117(1), 87-92 (2018). 